\title{
CLONING, SEQUENCING, AND IDENTIFICATION OF rhd $\alpha$-SUBUNIT GENE OF HALOAROMATIC DEHALOGENASE TERMINAL OXYGENASE FROM Pseudomonas aeruginosa LOCAL STRAIN
}

\author{
FAJRI IKHSAN and ENNY RATNANINGSIH \\ Biochemistry Division, Chemistry Study Program, Faculty of Mathematics and Natural Sciences, \\ Institut Teknologi Bandung, Ganesa 10 Street, Bandung 40132, Indonesia \\ ${ }^{*}$ E-mail: ennyratnaningsih1958@gmail.com
}

Accepted 27 April 2020, Published online 6 July 2020

\begin{abstract}
Haloaromatic is one of organohalogen pollutants found in the environment. These compounds are toxic, persistent, carcinogenic, and mutagenic. Accumulations of these compounds in the environment may cause serious diseases in organisms, including humans. Previous research results showed that breaking the bond of halogen atom from the aromatic ring could eliminate the toxicity of these compounds. Haloaromatic dehalogenase is an enzyme that possesses an ability to cut the halogen atom from the aromatic ring. In this research, a rhd $\alpha$-subunit gene of haloaromatic dehalogenase terminal oxygenase has been isolated and sequenced. The gene was isolated by PCR approach from Pseudomonas aeruginosa local strain, which is a Gramnegative bacterium found in soil as a pioneer growth bacterium. The sequence obtained indicated that rhd $\alpha$-subunit gene is 1290 bp in size. The RHD $\alpha$-subunit deduced amino acid has Cys98-X1-His100-X17-Cys118-X2-His121 and His217-X4His222-X149-Asp372 motifs, two important motifs in aromatic ring hydroxylating dioxygenase family, which is a large family of multi subunit and multifunctional proteins. One of its functions is in the breaking of halogen element from the aromatic carbon ring. Omori classification showed that RHD $\alpha$-subunit is classified as Group II of aromatic ring hydroxylating dioxygenase family, which includes haloaromatic dehalogenases. The result of 3D structure prediction also indicated that this protein belongs to this large family with 54 percentile Mol Probity score.
\end{abstract}

Key words: Pseudomonas aeruginosa, haloaromatic dehalogenases, rhd $\alpha$-subunit gene

\section{INTRODUCTON}

Haloaromatic is one group of many organohalogen pollutants found in the environment. These are also dominant pollutants ever found (Chaudry \& Chapalamadugu, 1991). Haloaromatics are salubrious compounds because of its toxic, persistent, carcinogenic, mutagenic, and accumulated behavior. There are many environmental pollutant haloaromatics, such as chlorotoluene (Pierce et al., 1983; Pierce et al., 1984), chlorobenzene (DeBont et al., 1986; Haigler et al., 1988), and chlorophenol (Apajalahti et al., 1986; Bruhn, 1988).

Dehalogenase is an enzyme that is able to release halogen from the organohalogen compounds. Some of the research results showed that the breaking of the halogen from the compounds could eliminate the toxicity of the organohalogen.

\footnotetext{
* To whom correspondence should be addressed.
}

Dehalogenase that is able to break the bond of the halogen atom from the aromatic carbon ring is called haloaromatic dehalogenase (Pankaj et al., 2014; Zhishu et al., 2017). Most haloaromatic dehalogenase work by dihydroxylation reaction, that is the addition of two $\mathrm{OH}$ groups to aromatic ring using the energy of a redox reaction. Most of the haloaromatic dehalogenases are included in a huge family of enzyme called aromatic ring hydroxylating dioxygenase.

Enzymes catalyzing the dihydroxylation reaction typically consist of two or three subunits with its specific function. Three components system usually consist of reductase, ferredoxin, and terminal oxygenase (Singleton, 2012). Terminal oxygenase consists of $\alpha$ - and $\beta$-subunits, where the $\alpha$-subunit is the most important part, as it not only has the catalytic site but also has a specific sequence that is used as a recognition area in enzyme classification. 


\section{MATERIALS AND METHODS}

\section{Bacterial strains and plasmid}

The bacterium used as a gene source was Pseudomonas aeruginosa local strain obtained from Microbiology Laboratory, SITH, Institut Teknologi Bandung. The E. coli TOP10 was used as the host cell in the cloning process, utilizing pGEM-T Easy as the cloning vector (Promega).

\section{Chromosome isolation and amplification of $r h d \alpha-$ subunit}

The chromosome of Pseudomonas aeruginosa local strain was isolated according to Klijn method. The three solutions used in this method were solution I ( $5 \mathrm{mg}$ lysozym in $500 \mu \mathrm{L}$ of $0.1 \mathrm{M}$ Tris$\mathrm{Cl}$ buffer at $\mathrm{pH} 8)$, solution II $(40 \mu \mathrm{L}$ of $10 \%(\mathrm{w} / \mathrm{v})$ SDS, $80 \mu \mathrm{L}$ of $0.5 \mathrm{M}$ EDTA $\mathrm{pH} 8,8 \mu \mathrm{L}$ of $20 \mathrm{mg} /$ $\mathrm{mL}$ proteinase $\mathrm{K}$, and $72 \mu \mathrm{L} \mathrm{ddH} 2 \mathrm{O}$ ), solution III ( $30 \mathrm{~mL}$ of $5 \mathrm{M} \mathrm{CH}_{3} \mathrm{COOK}, 5,75 \mathrm{~mL}$ of glacial acetic acid, and 14,25 mL ddH2O). The isolated chromosome was used as a template in PCR for the amplification of a rhd $\alpha$-subunit gene, using ATGGACGTCACTTCCACCCTGAGT and TCAG CTGCCGGCCACCTT as the forward and reverse primers. The experiment was performed in $20 \mu \mathrm{L}$ PCR cocktail that composed of $7.6 \mu \mathrm{L}$ ddH2O, 0.8 $\mu \mathrm{L}$ template, $0.8 \mu \mathrm{L}$ forward primer, $0.8 \mu \mathrm{L}$ reverse primer, and $10 \mu \mathrm{L}$ ready mix PCR (KAPA Taq Extra Hot Start Ready Mix, KAPA Biosystem). The amplification was carried out for 30 cycles, which consists of 5 minutes denaturation at $95^{\circ} \mathrm{C}, 30$ seconds of annealing at $55^{\circ} \mathrm{C}$, and 1 minute of elongation at $72^{\circ} \mathrm{C}$.

\section{Cloning of the rhd $\alpha$-subunit}

The obtained amplicon was cloned into the pGEM-T Easy vector according to Promega standard protocol. The recombinant plasmid carrying the rhd $\alpha$-subunit, called pGEM-rhd, was used to transform the E. coli TOP10 by heat shock method, and the positive transformants were selected by blue white screening $\alpha$-complementation (Sambrook, 2001). The transformants were cultured at $37^{\circ} \mathrm{C}$ on Lauria Bertani agar medium containing $100 \mu \mathrm{g} / \mathrm{mL}$ ampicillin, $100 \mu \mathrm{M}$ IPTG, and $50 \mu \mathrm{g} / \mathrm{mL}$ X-gal. The $p$-rhd recombinant plasmid was isolated from the white colonies by alkaline lysis according to Sambrook protocol (Sambrook, 2001), which was then sequenced and analyzed.

\section{Characterization and 3D structure prediction of RHD $\alpha$-subunit}

The RHD $\alpha$-subunit protein was deduced from the rhd $\alpha$-subunit cloned gene sequence. Characterization of the RHD $\alpha$-subunit was aimed to confirm that the rhd $\alpha$-subunit was part of the terminal oxygenase of a haloaromatic dehalogenase.
The first approach was searching for Cys-X1-HisX15-17-Cys-X2-His and His-X3-6-His-X148-149Asp motifs (Dong, 2005) to confirm that the RHD á-subunit was an enzyme that belongs to aromatic ring hydroxylating dioxygenase family. The second approach was applying Omori classification system (Nam et al., 2001), where haloaromatic dehalogenase is put in group II based on amino acid sequence homology of the $\alpha$-subunit terminal oxygenase of aromatic ring hydroxylating dioxygenase family. Two softwares used in this characterization were BioEdit and Mega 6. The 3D structure of RHD $\alpha$-subunit was established using I-Tasser online software (http://zhanglab.ccmb.med. umich.edu/I-TASSER/) and some of the suggested structures were validated using Mol Probity online software (http://rutile.biochem.duke.edu/).

\section{RESULTS AND DISCUSSION}

\section{Amplification, cloning, and sequencing of the rhd $\alpha$-subunit gene}

The rhd $\alpha$-subunit amplified from the Pseudomonas aeruginosa local strain chromosome appeared to be about $1300 \mathrm{bp}$ in size as shown by the agarose gel electrophoregram visualized under the UV light. The pGEM-rfd recombinant clone isolated from Escherichia coli TOP10 transformants was confirmed by re-PCR, which then sequenced using T7 and SP6 universal primers carried by the vector. The obtained sequence of the cloned rhd $\alpha$-subunit is shown in Figure 1, which revealed to be 1290 bp in size.

\section{Alignment of $r h d \alpha$-subunit and characterisation of RHD $\alpha$-subunit}

The cloned rhd $\alpha$-subunit gene showed high homology to genes of Pseudomonas aeruginosa with $99 \%$ identity. The deduced RHD $\alpha$-subunit also showed high homology to some Pseudomonas aeruginosa protein particularly to Gbc A protein, ring hydroxylating $\alpha$-subunit, and Rieske (2Fe-2S) protein. According to the official homepage of Pseudomonas Genome Database, GbcA protein of Pseudomonas aeruginosa is a ring hydroxylating dioxygenase protein predicted to have the same function with anthranilate dioxygenase, a protein with haloaromatic dehalogenase activity (Tsoi et al., 1999).

Further characterization of RHD $\alpha$-subunit indicated that the RHD $\alpha$-subunit could be classified into Group II based on Omori classification. The motif of Cys-X1-His-X15-17-Cys-X2-His appeared at Cys98-X1-His100-X17-Cys118-X2-His121 and the motif of His-X3-6-His-X148-149-Asp appeared at His217-X4-His222-X149-Asp372. The Xn indicated number of amino acids that separated the 


\begin{abstract}
$>$ rhd $\alpha$-subunit A
TGGACGTCACTTCCACCCTGAGTCTCGGCGATCCCCTGGAACCGGCCCGCAAGGCCACCGCCGACATGCTG CGCAGCCGCGACCACAGCTTCTCGCTGCCGCAGCCCTTCTACTGCGACCAGCGCCTGTTCGAGATCGACAT GCAGGAGATCTTCCACAAGGAATGGCTGATCGCCGGCATGACCTGCGAGATTCCCGCCAAGGGCAATTTC CTCACCCTGCAGATCGGCAAGAACCCGGTCCTGGTGATCCGTGGCGCCGAAGGCCAGGTGCATGCCTTCC ACAATGTCTGCCGGCACCGCGGCTCGCGCCTGTGCGTGAGCGAGAAAGGCAAGGTGGCCAAGCTGGTCT GCCCCCACCACCAGTGGACCTACGAGCTGGATGGCCGCCTGCTGTTCGCCGGCACCGAGATGGGCGCCG ACTTCGACATGAAGGAGTACGGCCTGAAGCCGATCCAGGTGAAGACCGCCGGCGGCTACATCTTCATCA GCCTGGCGGAGAACCCGCCGGCCATCGACGACTTCCTGGCGACGCTCGAGCACTACATGGAGCCGTACG ACATGGAGAACGCCAAGGTGGCGGTGCGGACCACCATCCGCGAAGCCGCCAACTGGAAGCTGGTGATC GAGAACAACCGCGAGTGCTACCACTGCAACGGCTCCCACCCGGAACTGCTGAAGACCCTGCTGGAGTG GGACGACGTCACCGACCCGCGCGCCAGCCAGGCGTTCAAGGACCAGGTGGCGGCCTGCACCAGCGCCT GGGACGCCGAGAAGATCCCGTACGCCCATGCCAGCTTCGGCCTGCGCAATCGCATCGTGCGCATGCCGC TGCTGGACGGCACGGTGTCGATGACCATGGACGGCAAGCAGGGCAGCAAGAAGCTCATGGGCCGGATC AAGAATCCCGACCTCGGCTCGATGCGCATCCTGCACCTGCCGCACTCCTGGAACCACTGCATGGGCGACC ACCTGATCGTCTTCACCGTGTGGCCGATCAGCGCCCAGGAAACCCTGGTCACCACCAAATGGCTGGTGCA CAAGGACGCGGTGGAAGGCGTCGACTACGACGTGGCGCGCCTGCGCGAGGTATGGGACGCGACCAACG ACCAGGATCGTCGGCTGGCCGAGGAGAACCAGCGCGGGATCAACTCCGACGCCTACCAGCCTGGTCCAT ACTCGAAGACCTACGAGTTCGGTGTGATCAATTTCCTCGACTGGTACAGCGAGCGCATGCTGAACAACCT CGGCGAGGAGTCCGCCCACGTGCGCAAGGTGGCCGGCAGCTGA
\end{abstract}

Fig. 1. The nucleotide sequence of the rhd $\alpha$-subunit.

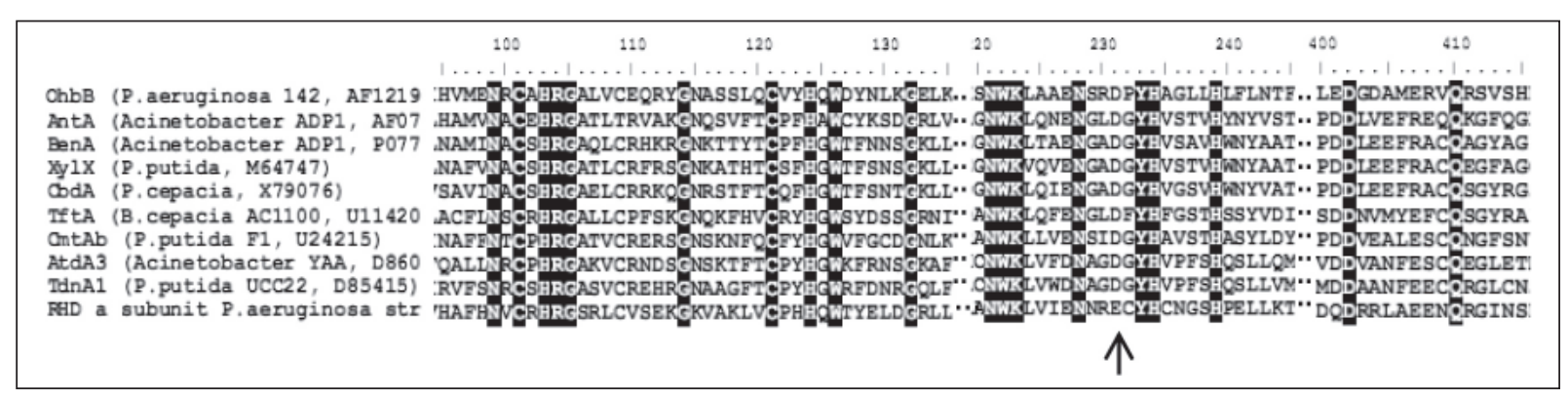

Fig. 2. The alignment of RHD $\alpha$-subunit with some subunit $\alpha$-subunit proteins of terminal oxygenase from Group II.

two residues in the motif. Cys98, His100, Cys118, and His 121 residues were the binding sites of Rieske iron sulphur cluster $(2 \mathrm{Fe}-2 \mathrm{~S})$ of RHD $\alpha$-subunit of Pseudomonas aeruginosa local strain. On the other hand, His217, His222, and Asp372 residues were the binding sites of $\mathrm{Fe}$ atom, which is the redox centre of dihydroxylation reaction. The appearance of these two amino acid sequence motifs on the RHD $\alpha$-subunit suggested that this protein was included into the aromatic ring hydroxylating dioxygenase family.

The result of protein alignment of RHD $\alpha$ subunit with some $\alpha$-subunit proteins of a terminal oxygenase component from Group II, showed the presence of conserved areas of proteins as depicted in Figure 2. Every single group of the Omori classification has different specific pattern and this pattern was the specific pattern of the Group II family.

Previous research stated that there is a negatively charge amino acid residue in the region of about two amino acid before His217, which is the
Fe binding site (Kauppi et al., 1998). In most bacteria, this negative residue is Asp. However, the amino acid number 214 in the deduced RHD $\alpha$ subunit was Glu (mark with $\uparrow$ in Figure 2), though the other residues that are conserved in aromatic ring hydroxylating dioxygenase namely Asn-TrpLys are still present. These residues were considered as the residues connecting $\alpha$-subunit and $\beta$-subunit in RHD $\alpha$-subunit. In Omori classification, the main characteristic is the existance of seven amino acids between Asn-Trp-Lys conserved residues and the first His of Fe binding site. As shown in Figure 5, these seven amino acids were present in the RHD $\alpha$-subunit. Thus, it is evident that the RHD $\alpha$ subunit is included in Group II, which also includes the haloaromatic dehalogenase.

Classification of RHD $\alpha$-subunit into Group II could be seen further in the constructed phylogenetic tree based on the homology of $\alpha$-subunit amino acid sequences of terminal oxygenase from aromatic ring hydroxylating family, as shown in Figure 3. This phylogenetic tree was established using three 


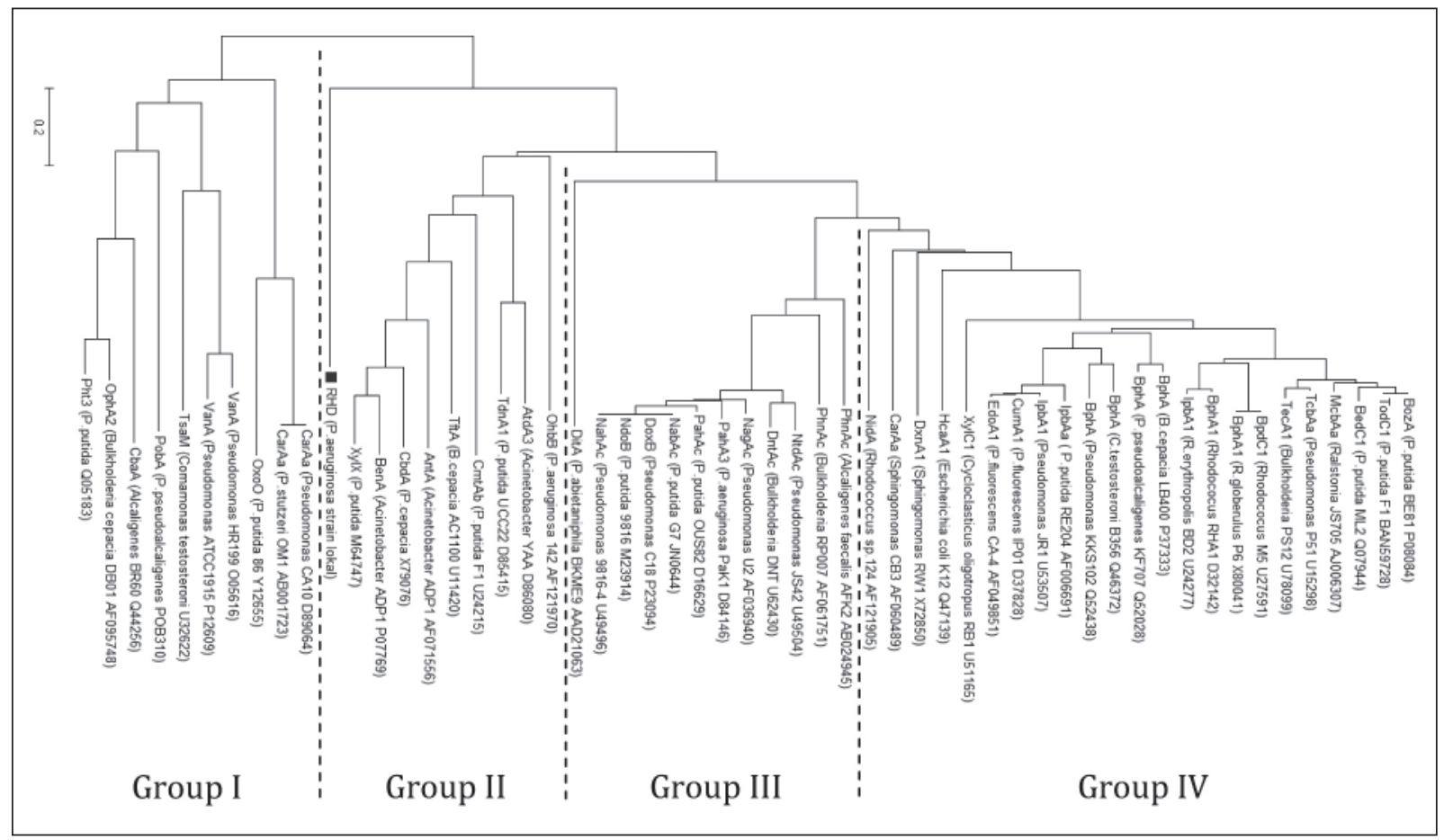

Fig. 3. Phylogenetic tree of the RHD $\alpha$-subunit with other $\alpha$-subunit proteins from aromatic ring hydroxylating dioxygenase family.

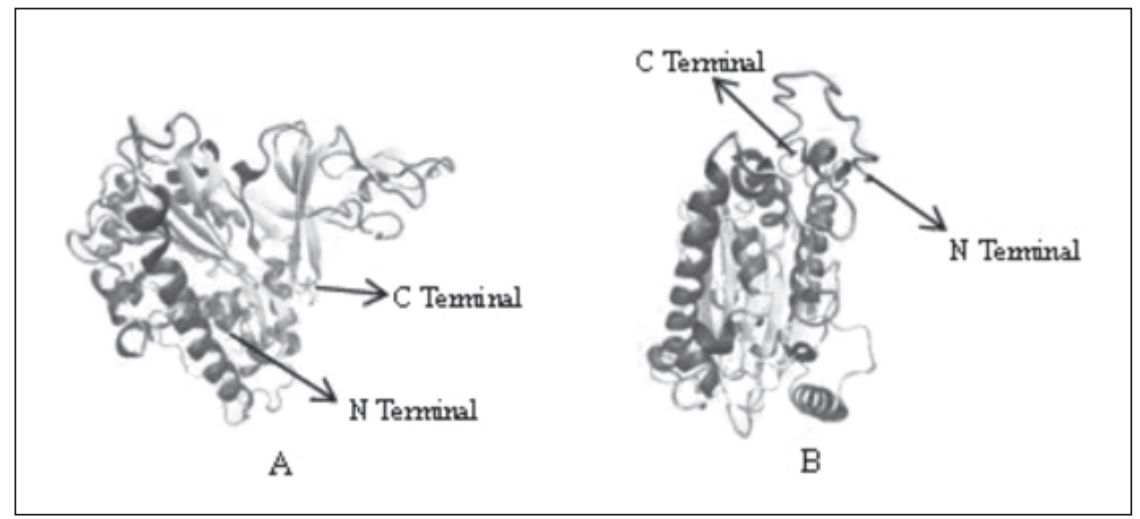

Fig. 4. Predicted structure of the RHD $\alpha$-subunit (A) Left side, (B) Front side.

different methods, neighbor joining, maximum likelihood, and minimum evolution. The result of these three methods clearly indicated that the RHD $\alpha$-subunit is in Group II of Omori classification.

\section{Prediction of the RHD $\alpha$-subunit 3D structure}

The 3D structure of the RHD $\alpha$-subunit was predicted using I-Tasser. Structures which were suggested by I-Tasser were validated using an online program called Mol Probity. This prediction aimed to check the homology of the RHD $\alpha$-subunit 3D structure with several published structures proteins in Protein Data Bank to clarify the classification and the function of the RHD $\alpha$ subunits.
I-Tasser is an online program that could predict the $3 \mathrm{D}$ structure of a protein. This program was based on the comparison of the amino acid sequence of a sample with other similar published 3D structures proteins, segment by segment. In the case of $3 \mathrm{D}$ protein structure prediction, there are two common methods, which are the comparison of the amino acid sequence of a sample with a single template, and the comparison of the amino acid sequence of a template with several templates. ITasser is based on the second method.

There were five structures that were suggested by I-Tasser, and the structure represented in Figure 4 is the best structure based on Mol Probity result. This structure has 54 percentile Mol Probity 
Table 1. Validation of the predicted RHD $\alpha$-subunit structure by MolProbity

\begin{tabular}{|c|c|c|c|c|}
\hline \multirow{2}{*}{$\begin{array}{l}\text { All-Atom } \\
\text { Contacts }\end{array}$} & Clashscose, all atems: & \multicolumn{2}{|l|}{2.52} & $98^{\mathrm{t}}$ percentile ${ }^{\prime}(\mathrm{N}=1784$, all resolutions) \\
\hline & \multicolumn{4}{|c|}{ Clashsoore is the number of senious steric overlaps $(>0.4 \mathrm{~A})$ per 1000 atoens. } \\
\hline \multirow{7}{*}{$\begin{array}{l}\text { Protein } \\
\text { Geometry }\end{array}$} & Poor rotamers & 28 & $7.63 \%$ & Goal: $<1 \%$ \\
\hline & Ramachandran outliers & 21 & $492 \%$ & Goal: $<0.05 \%$ \\
\hline & Ramachandran frovered & 360 & $8431 \%$ & Goal: $>98 \%$ \\
\hline & MolProbity scote & 2.38 & & $54^{\text {th }}$ percentile $(\mathrm{N}=27675,0 \AA-99 \AA)$ \\
\hline & CP deviations $>0.25 \AA$ & 18 & $4.49 \%$ & Goal: 0 \\
\hline & Bad backbone bonds: & $0 / 1715$ & $0,00 \%$ & Goal: $O \%$ \\
\hline & Bad backbone angles: & $4 / 2142$ & $0.19 \%$ & Goal: $<0.1 \%$ \\
\hline
\end{tabular}

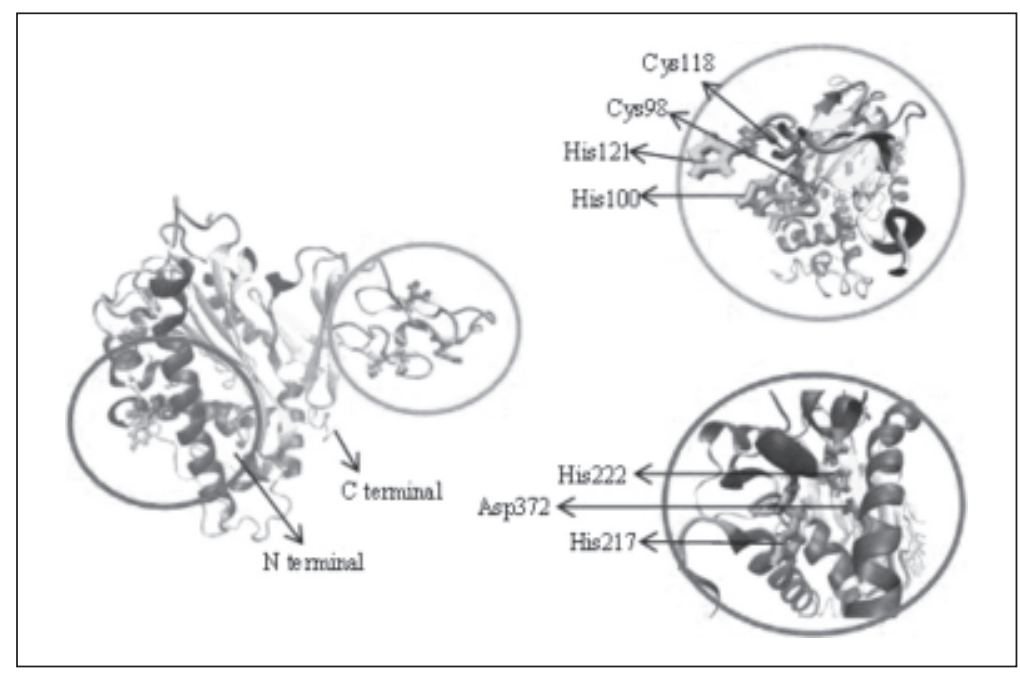

Fig. 5. The binding site of iron sulphur cluster $[2 \mathrm{Fe}-2 \mathrm{~S}]$ (blue circle) and the binding site of Fe atom (red circle).

score (Table 1). The Mol Probity score indicated the quality of the suggested structure, which was analyzed by comparing the determined structure with the published structures, where the highest score is $100 \%$. Therefore, the predicted RHD $\alpha$ subunit structure is a tolerable structure.

This structure has high homology with several proteins from aromatic ring hydroxylating dioxygenase family that has been published in Protein Data Bank. Some proteins that have high homology with the predicted RHD $\alpha$-subunit structure are ring hydroxylating dioxygenase from $S$. meliloti 1021, naphthalene 1,2-dioxygenase from Rhodococcus sp., and biphenyl dioxygenase from C. testosteroni. Unfortunately, among those proteins, there is no haloaromatic dehalogenase, probably because there is no published $3 \mathrm{D}$ structure of haloaromatic dehalogenase.

The predicted RHD $\alpha$-subunit structure has a binding site of Rieske iron sulphur cluster [2Fe-2S] which appeared as Cys98-X1-His100-X17-Cys118$\mathrm{X} 2$-His 121 on its primary structure. This binding site has a position on the head of RHD $\alpha$-subunit. The predicted RHD $\alpha$-subunit also has a binding site of Fe atom, which appeared as His217-X4-His222X149-Asp372 on its primary structure (Figure 5).
This binding site has a position at the back of RHD $\alpha$-subunit. Those parts appear on all proteins of aromatic ring hydroxylating dioxygenase family (Kauppi et al., 1998; Ferraro et al., 2005). At this predicted structure, there is a space under the binding site of Fe atom (Figure 6). This space is the catalytic pocket of the enzyme from aromatic ring hydroxylating dioxygenase family. Based on the results it can be concluded that the isolated rhd $\alpha$ subunit cloned is encoding for the aromatic dehalogenase of Pseudomonas aeruginosa.

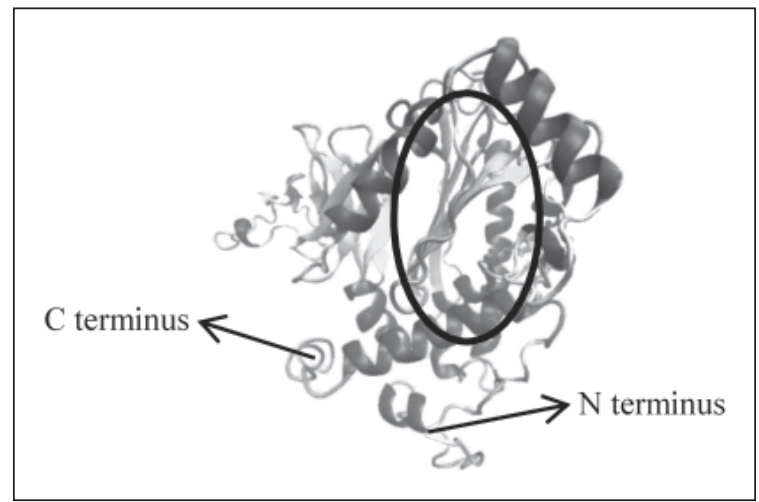

Fig. 6. Catalytic pocket of the RHD $\alpha$-subunit. 


\section{ACKNOWLEDGEMENTS}

This study was supported by the Faculty of Mathematics and Natural Sciences, Institut Teknology Bandung, Indonesia, through the Chemistry Study Program research fund for magister students.

\section{REFERENCES}

Apajalahti, J.H.A. \& Salonen, M.S.S. 1986. Degradation of polychlorinated phenols by Rhodococcus chlorophenolicus, Appl. Microbiol. Biotechnol., 25: 62-67.

Bruhn, C. \& Knackmuss, H.J. 1988. The in vivo construction of 4-chloro-2-nitrophenol assimilatory bacteria, Arch. Microbiol., 150: 171-177.

Chaudhry, G.R. \& Chapalamadugu, S. 1991. Biodegradation of Halogenated Organic Compounds, Microbiol. Rev., 55(1): 59-79.

DeBont, J.A., Vorage, M.J., Hartmans, S. \& Van den Tweel, W.J. 1986. Microbial degradation of 1,3dichlorobenzene, Appl. Environ. Microbiol., 52: 677-680.

Dong, X., Fushinobu, S., Fukuda, E., Terada, T., Nakamura, S., Shimizu, K., Nojiri, H., Omori, T., Shoun, H. \& Wakagi, T. 2005. Crystal Structure of the Terminal Oxygenase Component of Cumene Dioxygenase from Pseudomonas fluorescens IP01, J. Bacteriol., 187: 2483-2490.

Ferraro, D.J., Brown, E.N., Yu, C.L., Parales, R.E., Gibson, D.T. \& Ramaswamy, S. 2005. Structural investigation of the ferredoxin and terminal oxygenase components of the biphenyl 2,3dioxygenase from Sphingobium yanoikuyae B1, Biochem. Biophys. Res. Commun., 388: 175-190.

Haigler, B.E., Nishino, S.F. \& Spain, J.C. 1988. Degradation of 1,2-dicholorobenzene by a Pseudomonas sp, Appl. Environ. Microbiol., 54: 294-301.

Kauppi, B., Lee, K., Carredano, E., Parales, R.E., Gibson, D.T., Eklund, H. \& Ramaswamy, S. 1998. Structure of an aromatic-ringhydroxylating dioxygenase-naphthalene 1,2dioxygenase, Structure, 6(5): 571-586.
Klijn, N., Weerkamp, A.H. \& de Vos, V.M. 1991. Identification of mesophilic lactic acid bacteria by using polymerase chain reaction-amplified variable regions of 16S rRNA and specific DNA probes, Appl. Environ. Microbiol., 57: 33903393.

Nam, J.W., Nojiri, H., Yoshida, T., Habe, H., Yamane, H. \& Omori, T. 2001. New Classification System for Oxygenase Components Involved in RingHydroxylating Oxygenations, Biosci. Biotechnol. Biochem, 65: 254-263.

Pierce, J.E., Robinson, J.B. \& Colaruotolo, J.R. 1983. Substrate diversity of Pseudomonas spp. Containing chlorotoluene degradative plasmids, Dev. Ind. Microbiol., 24: 499-507.

Pierce, J.E. \& Chaplamandugu, S. 1984. Biodegradation of Halogenated Organic Compounds, Dev. Ind. Microbiol, 25: 597-602.

Pankaj, K.A. \& Hanhong, B. 2014. Role of Dehalogenases in Aerobic Bacterial Degradation of Cholorinated Aromatic Compounds, $J$. Chem., 2014: 1-10.

Sambrook, J. \& Russell, D.W. 2001. Molecular Cloning: A Laboratory Manual, $3^{\text {rd }}$ Ed., New York Cold Spring Harbor Laboratory Press).

Singleton, D.R., Hu, J. \& Aitken, M.D. 2012. Heterologous Expression of Polycyclic Aromatic Hydrocarbon Ring-Hydroxylating Dioxygenase Genes from a Novel PyreneDegrading Betaproteobacterium, Appl. Environ. Microbiol, 78(10): 3552-3559.

Tsoi, T.V., Plotnikova, E.G., Cole, J.R., Guerin, W.F., Bagdasarian, M. \& Tiedje, J.M. 1999. Cloning, Expression, and Nucleotide Sequence of the Pseudomonas aeruginosa 142 ohb Genes Coding dor Oxygenolytic ortho Dehalogenation of Halobenzoates, Appl. Environ. Microbio., 65 : 2151-2162.

Zhishu, L., Li, G. \& An, T. 2017. Purifying, cloning and characterizing a novel dealogenase from Bacillus sp. GZT to enchance the biodegradation of 2,4,6-tribromophenol in water, Environ. Pollut, 225: 104-111. 